\title{
On spirals and fixed point property
}

\author{
by
}

Roman Mańka (Warszawa)

\begin{abstract}
We study the famous examples of G. S. Young [7] and R. H. Bing [2]. We generalize and simplify a little their constructions. First we introduce Young spirals which play a basic role in all considerations. We give a construction of a Young spiral which does not have the fixed point property (see Section 5). Then, using Young spirals, we define two classes of uniquely arcwise connected curves, called Young spaces and Bing spaces. These classes are analogous to the examples mentioned above. The definitions identify the basic distinction between these classes. The main results are Theorems 4.1 and 6.1.
\end{abstract}

1. Introduction. All spaces are assumed to be metrizable. A space $X$ has the fixed point property iff for every mapping $f: X \rightarrow X$ there is a point $x_{0} \in X$ such that $f\left(x_{0}\right)=x_{0}$ (by a mapping we mean a continuous function). A classical result of dimension theory says that every mapping from a closed subset of a 1-dimensional space $X$ into the 1-sphere $S^{1}$ can be extended onto the whole space (see e.g. [3], Th. 3.2.10). Therefore if $X$ has the fixed point property, then $X$ contains no topological circle. Thus we have

Proposition. Let $X$ be a 1-dimensional arcwise connected space. If $X$ has the fixed point property, then $X$ is uniquely arcwise connected i.e. for each pair of distinct points $x, y \in X$ there exists a unique arc in $X$ from $x$ to $y$, denoted by $x y$ ).

The converse is not true. An important example was constructed by G. S. Young in 1960 (see [7]). Since that time, for topologists working in this area, it has been clear that Young's example required a more detailed study (several unproved claims about this example can be found in [2] and [4]). For this reason we undertake such a study in this paper.

If $D$ is a space homeomorphic to the unit closed $n$-ball $B^{n}$ in $\mathbb{R}^{n}$, then int $D$ denotes the subset of $D$ corresponding to the interior of $B^{n}$, and by $\partial D$ we denote the subset of $D$ corresponding to the boundary of $B^{n}$ in $\mathbb{R}^{n}$ (= the unit $(n-1)$-sphere $\left.S^{n-1}\right)$.

1991 Mathematics Subject Classification: Primary 54F50, 54H25. 
2. Rays. By a ray we mean here a uniquely arcwise connected space $P$ which can be obtained as the image of a surjective one-to-one mapping $\varphi:[0, \infty) \rightarrow P ; \varphi$ is called a parametrization of $P$. Such a mapping induces a linear order $\leq_{P}$ on $P$ corresponding to the natural order of the reals. Then $\varphi(0)$ is the smallest point in $P$ and it is also denoted by $o_{P}$; we call it the origin of $P$ (these notions are independent of the choice of a parametrization: if $\psi$ is another parametrization of $P$ then there is a homeomorphism $h$ : $[0, \infty) \rightarrow[0, \infty)$ such that $\psi=\varphi \circ h$. For $a \in P$ we define

$$
P(a)=\left\{x \in P: a \leq_{P} x\right\} .
$$

Thus $P=P\left(o_{P}\right)$. It is clear that $P(a)$ is a ray; we call it the subray of $P$ determined by $a$. If $P$ is a subset of a space $X$ then we define the limit set of $P($ in $X$ ) by the formula

$$
L(P)=\bigcap_{a \in P} \overline{P(a)} .
$$

It is also the limit set of every subray of $P$. Clearly $\bar{P}=P \cup L(P)$.

We deal with very special rays - topological half-lines, i.e. spaces homeomorphic to $[0, \infty)$.

2.1. Proposition. If $P$ is a topological half-line in a space $X$, then:

(1) $P \cap L(P)=\emptyset$,

(2) if $L(P)$ contains at least two points then $\bar{P}$ is locally connected at $x \in \bar{P}$ iff $x \in P$,

(3) $\bar{P}$ is irreducible between $o_{P}$ and any point $x_{0} \in L(P)$, i.e. no proper closed connected subset of $P$ contains both $x_{0}$ and $o_{P}$ (cf. [5]).

Proof. (1) simply follows from the fact that $\varphi^{-1}(C)$ is compact for a compact set $C \subset P$. (2) trivially follows from (1).

(3) simply follows from (1) and the fact that every point $x \in P-\left\{o_{P}\right\}$ separates $\bar{P}$ between $o_{P}$ and $L(P)$.

It is a standard fact that if $X$ is a uniquely arcwise connected space, then every point $x_{0} \in X$ determines a partial order $\leq_{x_{0}}$ on $X$ given by the formula

$$
x \leq_{x_{0}} y \equiv x_{0} x \subset x_{0} y .
$$

As usual, $x<_{x_{0}} y \equiv\left(x \leq_{x_{0}} y\right.$ and $\left.x \neq y\right)$. In the sequel we use the following

2.2. Proposition [6]. Let $X$ be as above and let $f: X \rightarrow X$ be a mapping. If $f\left(x_{0}\right) \neq x_{0}$ for some $x_{0} \in X$, then there exists a unique ray $P$ in $X$ satisfying the following conditions:

(1) $o_{P}=x_{0}$

(2) $p<_{x_{0}} f(p)$ for every $p \in P$, and $P$ is maximal with respect to this property, 
(3) $P \cap p f(p)$ is an arc for every $p \in P$,

(4) if there is a point $p_{0} \in P$ such that no 3 -od in $X$ has its vertex on $P\left(p_{0}\right)$ (三 no point of $P\left(p_{0}\right)$ is a branch point of $\left.X\right)$, then $f(L(P))=L(P)$.

3. Young spirals. By a double Warsaw circle we mean a compact space $W=\bar{A}_{0} \cup \bar{A}_{1}$, where $A_{0}, A_{1}$ are disjoint topological half-lines in $W$ such that $L\left(A_{0}\right), L\left(A_{1}\right)$ are disjoint arcs, $A_{0} \cap L\left(A_{1}\right)=\left\{o_{A_{0}}\right\}$ and $A_{1} \cap L\left(A_{0}\right)=\left\{o_{A_{1}}\right\}$. Clearly, $W$ is a continuum containing no circle, it has two arc components $A_{0} \cup L\left(A_{1}\right)$ and $A_{1} \cup L\left(A_{0}\right)$, and $W$ is not locally connected at $x$ iff $x \in$ $L\left(A_{0}\right) \cup L\left(A_{1}\right)$. We also have

3.1. Proposition. (1) Every proper subcontinuum of $W$ has the fixed point property.

(2) If a mapping $f: W \rightarrow W$ has no fixed point, then $f$ is surjective, $L\left(A_{1}\right)=f\left(L\left(A_{0}\right)\right)$ and $L\left(A_{0}\right)=f\left(L\left(A_{1}\right)\right)$.

Proof. (1) follows from classical results (see [2], Ths. 6 and 8). Thus the first assertion of (2) follows from (1). It remains to show the equalities.

One easily sees that $f\left(L\left(A_{0}\right)\right) \subset L\left(A_{0}\right) \cup L\left(A_{1}\right)$ (otherwise $f(W)$ would be arcwise connected, hence contained in one arc component of $W$, which is impossible by the initial part of the proof). We must have $f\left(L\left(A_{0}\right)\right) \subset L\left(A_{1}\right)$ since $L\left(A_{0}\right)$ is an arc. Then $f\left(A_{1}\right) \subset A_{0} \cup L\left(A_{1}\right)$, and consequently $f\left(A_{0}\right) \subset$ $A_{1} \cup L\left(A_{0}\right)$. Then $f\left(A_{0}(a)\right)$ is a subray of $A_{1}$ for some $a \in A_{0}$. Let $x_{1}<$ $x_{2} \leq \ldots$ be a cofinal sequence of $A_{0}(a)$. Since the $\overline{A_{0}\left(x_{n}\right)}$ are continua converging to $L\left(A_{0}\right), \overline{f\left(A_{0}\left(x_{n}\right)\right)}$ converges to $f\left(L\left(A_{0}\right)\right)$. But $L\left(A_{1}\right) \subset \overline{f\left(A_{0}\left(x_{n}\right)\right)}$ since $f\left(A_{0}\left(x_{n}\right)\right)$ is a subray of $A_{1}$ for every $n \geq 1$. It follows that $f\left(L\left(A_{0}\right)\right)=$ $L\left(A_{1}\right)$; the other equality follows by an analogous argument.

Remark. There exist uncountably many topological types of double Warsaw circles (see [1] for stronger results).

By a Young spiral we mean a continuum $Y$ such that $Y=\bar{S}$, where $S$ is a topological half-line with $L(S)$ being a double Warsaw circle (see Fig. 1).

With this notation we have

3.2. Proposition. (1) If a subcontinuum of $Y$ does not have the fixed point property, then it contains $L(S)$.

(2) If $f: Y \rightarrow Y$ is a fixed point free mapping, then $f(S) \subset S, x<_{S} f(x)$ for every $x \in S$, and $f(L(S))=L(S)$.

Proof. (1) follows from 3.1(1). In order to prove that $f(S) \subset S$, suppose, to the contrary, $f(S) \cap L(S) \neq \emptyset$. Since $f(S)$ is arcwise connected, $f(S)$ must be a subset of an arc component of $L(S)$. Then $f(Y)=f(S)$ would be a proper subset of $L(S)$, contrary to (1). This proves the inclusion. The rela- 


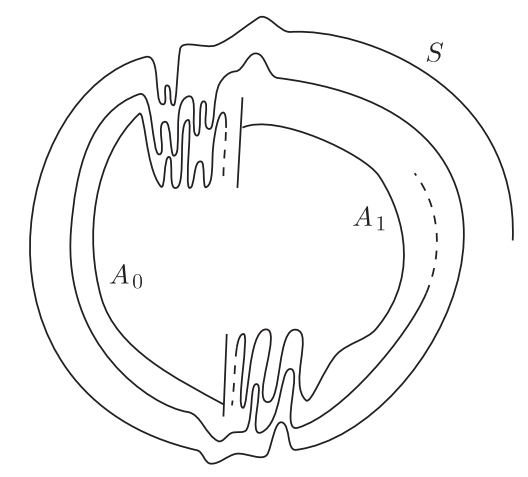

Fig. 1. A Young spiral

tion $x<_{S} f(x)$ easily follows. Consequently, $f(S(x)) \subset S(x)$ for every $x \in S$. It follows that $f(L(S)) \subset L(S)$, and the reverse inclusion follows from (1).

4. Young spaces. Let $S$ be a Young spiral with $L(S)=\bar{A}_{0} \cup \bar{A}_{1}$ a double Warsaw circle (see definition above). By a Young space we mean a uniquely arcwise connected continuum $X=\bar{S} \cup a_{0} a_{1}$ such that $a_{0} a_{1} \cap\left(A_{0} \cup L\left(A_{1}\right)\right)=$ $\left\{a_{0}\right\}, a_{0} a_{1} \cap S=\left\{o_{S}\right\}$ and $a_{0} a_{1} \cap\left(A_{1} \cup L\left(A_{0}\right)\right)=\left\{a_{1}\right\}$. Thus the arc $a_{0} a_{1}$ meets each arc component of $S$ in a single point (see Fig. 2).

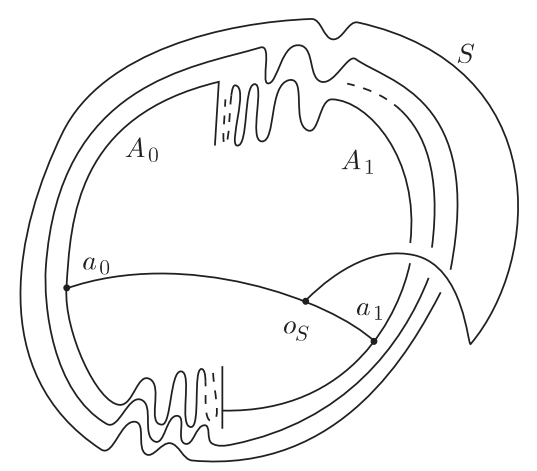

Fig. 2. A Young space

4.1. Theorem. A Young space $X=\bar{S} \cup a_{0} a_{1}$ has the fixed point property iff the Young spiral $\bar{S}$ does.

Pr o of. Suppose there is a fixed point free mapping $f: \bar{S} \rightarrow \bar{S}$. We shall show that $f$ can be extended to a fixed point free mapping $f^{*}: X \rightarrow X$. Hence we must define $f^{*}$ on $a_{0} a_{1}$. Note that $a_{0} a_{1}=a_{0} o_{S} \cup o_{S} a_{1}$ and the $\operatorname{arcs} a_{0} o_{S}, o_{S} a_{1}$ meet only in $o_{S}$. We have $f\left(o_{S}\right) \in S-\left\{o_{S}\right\}$ (see 3.2) and $f\left(a_{i}\right) \in A_{1-i} \cup L\left(A_{i}\right)$ (see 3.1 and 3.2). Define $f^{*}$ on $a_{i} o_{S}$ to be a 
homeomorphism transforming this arc onto the arc $f\left(a_{i}\right) f\left(o_{S}\right)$ (in this order $), i=0,1$. This gives a continuous extension of $f$ onto $X$. Note that $f\left(a_{i}\right) f\left(o_{S}\right)=f\left(a_{i}\right) a_{1-i} \cup a_{1-i} o_{S} \cup o_{S} f\left(o_{S}\right)$. Since $f\left(a_{i}\right) a_{1-i} \subset A_{1-i} \cup L\left(A_{i}\right)$, it follows that $o_{i} o_{S} \cap f^{*}\left(a_{i} o_{S}\right)=\left\{o_{S}\right\}$, hence $f^{*}$ has no fixed point.

Suppose that there is a fixed point free mapping $f: X \rightarrow X$. To get a contradiction, it suffices to show that $f$ maps $\bar{S}$ into itself. Let $x_{0}=o_{S}$. Since $f\left(x_{0}\right) \neq x_{0}$, by Proposition 2.2 there exists a ray $P$ in $X$ satisfying conditions (1)-(4) of that proposition. Since every ray in $X$ satisfies conditions from 2.2(4), we have $f(L(P))=L(P)$. It follows that $P=S$ (otherwise $L(P)$ is an arc or a point). By $2.2(2), x<_{o_{S}} f(x)$ for every $x \in P$. It follows that $f(S) \subset S$, which completes the proof.

5. Example. There exists a Young spiral $Y=\bar{S}$ without the fixed point property (see Fig. 3).

The construction is done in the plane $\mathbb{R}^{2}$. Let $\alpha: \mathbb{R}^{2} \rightarrow \mathbb{R}^{2}$ be the antipodal mapping $\alpha(x, y)=(-x,-y)$. The image $\alpha(Z)$ of a set (or a point) $Z$ will be sometimes also denoted by $Z^{\prime}$.

Let $g:(0,1] \rightarrow[1,3]$ be the mapping given by

$$
g(x)=2+\sin \frac{\pi}{x}
$$

and let $\Gamma$ denote the graph of $g$, i.e. $\Gamma=\{(x, g(x)): x \in(0,1]\}$. Set $a=(0,-3), b=(2,0), c=(1,2)$. Then $A=\overline{a b} \cup \overline{b c} \cup \Gamma$ is a topological half-line with $o_{A}=a$ (we denote by $\overline{u v}$ the line segment in $\mathbb{R}^{2}$ with end points $u, v)$. The set $\bar{A} \cup \overline{A^{\prime}}$ is a double Warsaw circle and $\alpha$ determines a fixed point free mapping on it.

Let $g_{n}:[0,1] \rightarrow[1,4], n=1,2, \ldots$, be mappings satisfying the conditions

(1) $g_{n}(0)=3+1 / n, g_{n}(1)=2+1 / n$,

(2) $g_{1}(x)>g_{2}(x)>\ldots$ for every $x \in[0,1]$,

(3) $g_{n}(x) \rightarrow g(x)$ as $n \rightarrow \infty$ for $x>0$,

(4) $\sup _{x \in[0,1]}\left|g_{n}(x)-g_{n+1}(x)\right| \rightarrow 0$ as $n \rightarrow \infty$.

Denote by $\Gamma_{n}$ the graph of $g_{n}$.

Remark. Note that such a sequence always exists: for instance,

$$
g_{n}(x)=3+\frac{1}{n}-\left(1-\sin \frac{\pi}{x}\right) x^{1 / n}
$$

satisfy the above conditions.

Set $a_{n}=(0,-3-1 / n), b_{n}=(2+1 / n, 0), c_{n}=(1,2+1 / n)$ and put

$$
S=\bigcup_{n=1}^{\infty}\left(\overline{a_{n} b_{n}} \cup \overline{b_{n} c_{n}} \cup \Gamma_{n} \cup \overline{a_{n}^{\prime} b_{n}^{\prime}} \cup \overline{b_{n}^{\prime} c_{n+1}^{\prime}} \cup \Gamma_{n+1}\right) .
$$




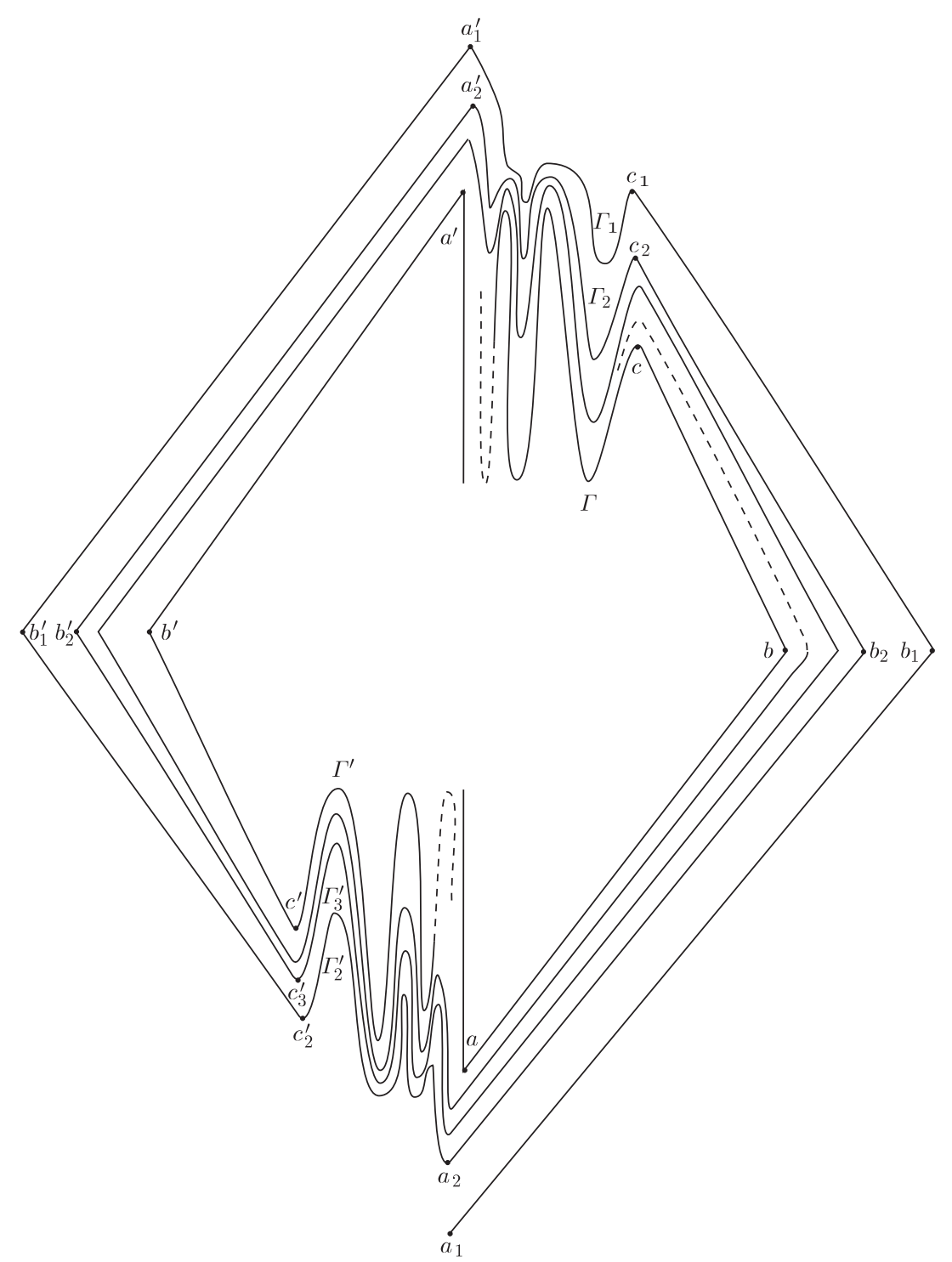

Fig. 3. A Young spiral without the fixed point property

Note that $S$ is a topological half-line and $Y=\bar{S}$ is a Young spiral with $L(S)=\bar{A} \cup \overline{A^{\prime}}$. We are going to prove that there is a fixed point free map- 
ping $f: Y \rightarrow Y$. We define $f$ as follows:

$$
f(p)= \begin{cases}\alpha(p) & \text { for } p \in \bar{A} \cup \overline{A^{\prime}} \cup \bigcup_{n=1}^{\infty}\left(\overline{a_{n} b_{n}} \cup \overline{a_{n}^{\prime} b_{n}^{\prime}} \cup \Gamma_{n+1}^{\prime}\right), \\ (1-t) b_{n}^{\prime}+t c_{n+1}^{\prime} & \text { for } p=(1-t) b_{n}+t c_{n} \in \overline{b_{n} c_{n}}, 0 \leq t \leq 1, \\ (1-t) b_{n}+t c_{n} & \text { for } p=(1-t) b_{n}^{\prime}+t c_{n+1}^{\prime} \in \overline{b_{n}^{\prime} c_{n}^{\prime}}, 0 \leq t \leq 1, \\ \left(-x,-g_{n+1}(x)\right) & \text { for } p=\left(x, g_{n}(x)\right) \in \Gamma_{n} .\end{cases}
$$

One easily checks that $f$ is well defined, fixed point free, and extends the antipodal map $\alpha$ restricted to $\bar{A} \cup \overline{A^{\prime}}$. It remains to prove that $f$ is continuous at every point $p_{0} \in Y$. The only nontrivial case is when $p_{0} \in L(A)$. So, let $p_{1}, p_{2}, \ldots$ be a sequence in $Y$ converging to $p_{0}$. We have to prove that $f\left(p_{i}\right) \rightarrow f\left(p_{0}\right)$ as $i \rightarrow \infty$. Since $f\left(p_{0}\right)=\alpha\left(p_{0}\right)$, it suffices to consider the case where $p_{i} \in \bigcup_{n=1}^{\infty} \Gamma_{n}$, i.e. $p_{i}=\left(x_{i}, g_{n_{i}}\left(x_{i}\right)\right)$. Then $n_{i} \rightarrow \infty$ as $i \rightarrow \infty$. We have to show that $f\left(p_{i}\right) \rightarrow \alpha\left(p_{0}\right)$ as $i \rightarrow \infty$. Since $\alpha\left(p_{i}\right) \rightarrow \alpha\left(p_{0}\right)$ it suffices to show that $\left|f\left(p_{i}\right)-\alpha\left(p_{i}\right)\right| \rightarrow 0$. But

$$
\begin{aligned}
\left|f\left(p_{i}\right)-\alpha\left(p_{i}\right)\right| & =\left|\left(-x_{i},-g_{n_{i}+1}\left(x_{i}\right)\right)-\left(-x_{i},-g_{n_{i}}\left(x_{i}\right)\right)\right| \\
& =\left|g_{n_{i}}\left(x_{i}\right)-g_{n_{i}+1}\left(x_{i}\right)\right|
\end{aligned}
$$

and by (4) these numbers tend to 0 , which completes the construction.

6. Bing spaces. By a Bing space we mean a uniquely arcwise connected continuum $X=\bar{S} \cup a_{0} o_{S}$, where $\bar{S}=S \cup \bar{A}_{0} \cup \bar{A}_{1}$ is a Young spiral (in its standard presentation), $a_{0} o_{S} \cap\left(A_{0} \cup L(A)\right)=\left\{a_{0}\right\}, a_{0} o_{S} \cap\left(A_{1} \cup L\left(A_{0}\right)\right)=L$ is a continuum and $a_{0} o_{S} \cap S=\left\{o_{S}\right\}$ (see Fig. 4). Let $a_{1}$ be the greatest point of $L$ (in the order of $a_{0} o_{S}$ from $a_{0}$ to $o_{S}$ ).

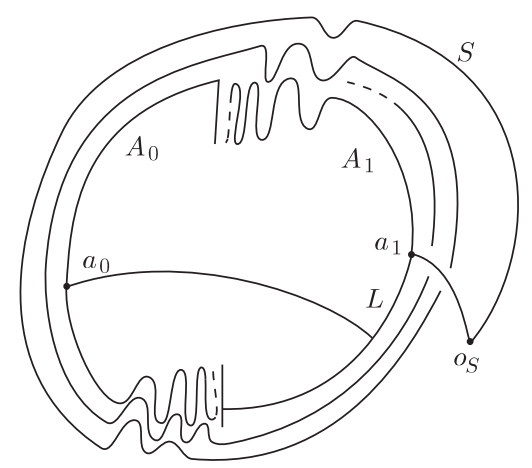

Fig. 4. A Bing space

6.1. Theorem (comp. [2]). Every Bing space $X$ has the fixed point property. There exists a Bing space $X_{0}$ and a 2-cell $D$ such that $D \cap X_{0}$ is an arc and $X_{0} \cup D$ does not have the fixed point property. Moreover, we can 
choose a decreasing sequence of discs $D \supset D_{1} \supset D_{2} \supset \ldots$ converging to a point such that for every $n, X_{0}$ and $D_{n}$ have the above property.

Proof. Let $f: X \rightarrow X$ be a mapping. We have to prove that $f$ has a fixed point. Suppose not.

Applying 2.2 we get a ray $P$ in $X$ emanating from $a_{1}$ with $f(L(P))=$ $L(P)$ and with $a_{1} f\left(a_{1}\right) \cap P$ being an arc. It follows that $P=a_{1} o_{S} \cup S$, hence $L(P)=L(S)=\bar{A}_{0} \cup \bar{A}_{1}$. Then $f\left(a_{1}\right) \in L(P)$. Since $a_{1} f\left(a_{1}\right)$ meets $P$ along an arc, we must have $f\left(a_{1}\right) \in P$. This is impossible since $P \cap L(P)=\emptyset$. It remains to prove the second part of the theorem.

To this end, take $\bar{S}$ to be a Young spiral without the fixed point property (see the Example). Define $X_{0}$ to be the Bing space $\bar{S} \cup a_{0} o_{S}$ in which $L=\left\{a_{1}\right\}$.

We claim that $X_{0}$ has the desired property. In order to prove this, choose arbitrary points $u \in \operatorname{int} a_{0} a_{1}$ and $v \in \operatorname{int} a_{1} o_{S}$. Attach a 2-cell $D$ to $X_{0}$ in such a way that $D \cap X_{0}=u v \subset \partial D$ (see Fig. 5).
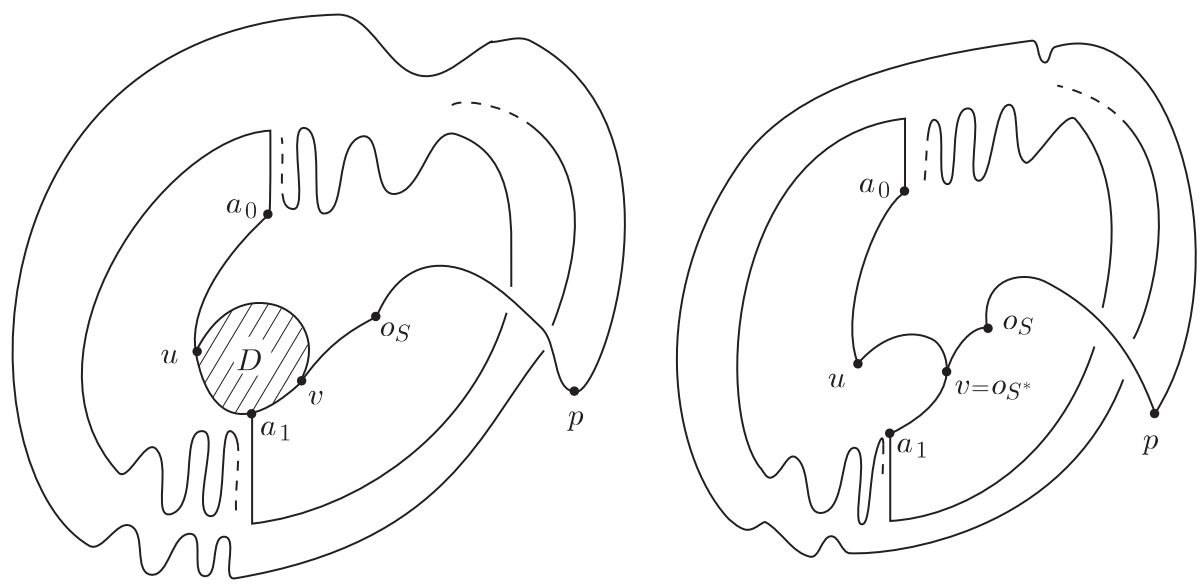

Fig. 5. Retraction of $X_{0} \cup D$ onto a Young space (only the arc $o_{S} p$ lies above the plane containing the rest of the constructed sets)

Let $(u v)^{*} \subset \partial D$ be the arc with endpoints $u$ and $v$ complementary to $u v$ in $\partial D$. Let $S^{*}=(u v)^{*} \cup v o_{S} \cup S$. Hence $S^{*}$ is a topological half-line with $o_{S^{*}}=u$. Since $\overline{S^{*}}$ is homeomorphic to $\bar{S}, \overline{S^{*}}$ is a Young spiral without the fixed point property. Hence, by 4.1 , the Young space $X_{1}=\overline{S^{*}} \cup a_{0} a_{1}$ does not have the fixed point property. We conclude that $X_{0} \cup D$ does not have the fixed point property since there exists a retraction of $X_{0} \cup D$ onto $X_{1}$. To complete the proof it remains to note that there is a decreasing sequence of discs in $D$ converging to $a_{1}$ such that each of them can play the role of $D$ in the above proof. 
Acknowledgments. I am grateful to Professors J. Krasinkiewicz and K. Sieklucki for discussions and stimulating remarks. Especially, I wish to express my gratitude to Professor K. Sieklucki for encouragement to elaborate on the general problems mentioned in the introduction, and to Professor J. Krasinkiewicz for enormous work leading to the final, significantly improved version of the whole paper and particularly Section 5 .

\section{References}

[1] M. M. Awartani, The fixed remainder property for self-homeomorphisms of Elsa continua, Topology Proc. 11 (1986), 225-238.

[2] R. H. Bing, The elusive fixed point property, Amer. Math. Monthly 76 (1969), 119-132.

[3] R. Engelking, Dimension Theory, PWN, Warszawa, and North-Holland, Amsterdam, 1978.

[4] W. Holsztyński, Fixed points of arcwise connected spaces, Fund. Math. 69 (1969), 289-312.

[5] K. Kuratowski, Topology, Vols. I and II, Academic Press, New York, and PWNPolish Scientific Publishers, Warszawa, 1966 and 1968.

[6] R. Mańka, On uniquely arcwise connected curves, Colloq. Math. 51 (1987), 227238.

[7] G. S. Young, Fixed-point theorems for arcwise connected continua, Proc. Amer. Math. Soc. 11 (1960), 880-884.

INSTITUTE OF MATHEMATICS

POLISH ACADEMY OF SCIENCES

ŚNIADECKICH 8

00-950 WARSZAWA, POLAND 